\title{
Magnetization plateaus in weakly coupled dimer spin system
}

\author{
Alexei K. Kolezhuk \\ Institut für Theoretische Physik, Universität Hannover, Appelstraße 2, D-30167 Hannover, Germany \\ Institute of Magnetism, National Academy of Sciences and Ministry of Education of Ukraine \\ 36(b) Vernadskii avenue, 252142 Kiev, Ukraine
}

(last modified September 15, 1998; Received May 10, 2018)

\begin{abstract}
I study a spin system consisting of strongly coupled dimers which are in turn weakly coupled in a plane by zigzag interactions. The model can be viewed as the strong-coupling limit of a twodimensional zigzag chain structure typical, e.g., for the $(a c)$-planes of $\mathrm{KCuCl}_{3}$. It is shown that the magnetization curve in this model has plateaus at $\frac{1}{3}$ and $\frac{2}{3}$ of the saturation magnetization, and an additional plateau at $\frac{1}{2}$ can appear in a certain range of the model parameters; the critical fields are calculated perturbatively. It is argued that for the three-dimensional lattice structure of the $\mathrm{KCuCl}_{3}$ family the plateaus at $\frac{1}{4}$ and $\frac{3}{4}$ of the saturation can be favored in a similar way, which might be relevant to the recent experiments on $\mathrm{NH}_{4} \mathrm{CuCl}_{3}$ by Shiramura et al., J. Phys. Soc. Jpn. 67, 1548 (1998).
\end{abstract}

75.10.Jm, 75.60.Ej, 75.50.Ee

\section{INTRODUCTION}

In the last couple of years there has been a growing interest to the phenomenon of magnetization plateaus in one-dimensional (1D) spin systems. 14 In a variety of models, the magnetization per site $m_{z}$ as a function of the applied magnetic field $H$ exhibits plateaus at certain values of $m_{z}$; at those plateaus the magnetization in units of the saturation $M=m_{z} / S$ (here $S$ is the spin of a magnetic ion) is "locked" at some rational number (at least, up to now no indications of the possibility to have plateaus at irrational $M$ were found). Oshikawa et al 1 have shown that in purely one-dimensional systems the allowed values of $M$, at which the plateaus are possible, are given by the following condition

$$
Q S(1-M) \in \mathbb{Z},
$$

where $Q$ is the number of magnetic ions in the magnetic elementary cell of the ground state (which can be different from the elementary cell prescribed by the Hamiltonian 1 ). This condition is necessary but not sufficient; in other words, if the plateau exists, it has to be at one of the values of $M$ determined by (1), but in principle the plateaus do not have to exist at all values of $M$ which are allowed by (11). Validity of the result (11) is supported by a considerable amount of numerical and analytical data, as well as by the recent experimental observation 1 of the $M=\frac{1}{2}$ plateau in the $S=$ 1 bond-alternating chains realized in $\left[\mathrm{Ni}_{2} \text { (Medpt }\right)_{2}(\mu-$ ox $\left.)\left(\mu-\mathrm{N}_{3}\right)\right] \mathrm{ClO}_{4} \cdot 0.5 \mathrm{H}_{2} \mathrm{O}$.

The present paper is motivated by the recent highfield magnetization measurements $\mathrm{D}^{\mathrm{B}}$ in the double-chain $S=\frac{1}{2}$ compound $\mathrm{NH}_{4} \mathrm{CuCl}_{3}$ which have revealed wellpronounced plateaus at $M=\frac{1}{4}$ and $M=\frac{3}{4}$ in the temperature range $T<1.5 \mathrm{~K}$. At room temperature, the crystal structure of this material coincides with that of $\mathrm{KCuCl}_{3}$, and the main feature is presence of the double chains of edge-sharing $\mathrm{CuCl}_{6}$ octahedra, separated by $\mathrm{NH}_{4}^{+}$ions (see figs. 2,3 of Ref. 16 or fig. 1 of Ref. 17). Double chains composed of magnetic $\mathrm{Cu}^{2+}$ ions can be viewed as spin- $\frac{1}{2}$ bond-alternating antiferromagnetic (AF) zigzag chains with two different nearest-neighbor exchange constants $J_{2}, J_{3}$ and next-nearest-neighbor exchange $J_{1}$ (see fig. 1 of Ref. 17, and Fig. 11 of the present paper); in what follows, the word "chain" is used in the meaning of a zigzag (double) chain. If one assumes that the essential physics in $\mathrm{NH}_{4} \mathrm{CuCl}_{3}$ is determined by single chains, then the appearance of plateaus at $\frac{1}{4}$ and $\frac{3}{4}$ of saturation means that due to a certain rather complicated spontaneous symmetry breaking the magnetic elementary cell contains $Q=8 \mathrm{Cu}^{2+}$ ions instead of $Q=2$ as suggested by the Hamiltonian symmetry; still, then absence of plateau at $M=\frac{1}{2}$ which is characteristic for a single chain 9 is puzzling.

Values of the exchange constants in $\mathrm{NH}_{4} \mathrm{CuCl}_{3}$, as well as in the other materials of the so-called $\mathrm{KCuCl}_{3}$ family, 1617 are presently not known. There are certain argumentst based on fitting the susceptibility data for the isostructural compound $\mathrm{KCuCl}_{3}$ which suggest that one of the nearest-neighbor couplings $\left(J_{3}\right)$ is dominating. 19 On the other hand, neutron scattering data for $\mathrm{KCuCl}_{3}$ strongly suggest that interchain interactions are important. 20 From the susceptibility data 1 . 15 one can guess that the intrachain couplings in $\mathrm{NH}_{4} \mathrm{CuCl}_{3}$ are much weaker than in $\mathrm{KCuCl}_{3}$ and thus interchain couplings should play even more important role; this assumption also fits well to the fact that the zero-field ground state of $\mathrm{NH}_{4} \mathrm{CuCl}_{3}$ is magnetic at low temperature 15

In the present paper I show that interchain couplings can considerably affect the number and positions of the plateaus. To demonstrate that, I consider a simple model describing a system of zigzag chains coupled in a plane; within the effective spinless fermion model valid in the limit of weakly coupled dimers it is shown that this model can exhibit magnetization plateaus at $\frac{1}{3}, \frac{1}{2}$, and $\frac{2}{3}$ of the saturation. The critical fields are calculated using 
the lowest-order perturbation theory around the "atomic limit" of the model. Physics of the plateau at $M=\frac{1}{2}$ is determined by single chains and is almost exactly the same as studied in Ref. 9, while the plateaus at $M=\frac{1}{3}, \frac{2}{3}$ appear solely due to the two-dimensional interchain couplings. Generally speaking, the number and positions of the "additional" plateaus caused by interchain interactions strongly depend on the topology of the specific lattice. Possible relevance of this mechanism to the experiments on $\mathrm{NH}_{4} \mathrm{CuCl}_{3}$ is discussed: it is argued that the three-dimensional lattice structure of $\mathrm{NH}_{4} \mathrm{CuCl}_{3}$ naturally favors the appearance of plateaus at $\frac{1}{4}$ and $\frac{3}{4}$ of the saturation. We also discuss possible reasons of absence of the other plateaus in this material.

The paper is organized as follows: in Sect. II the effective model for weakly coupled dimer system is introduced and the magnetization curve of the two-dimensional system of coupled chains is studied, Sect. III is devoted to the analysis of characteristic features of threedimensional coupled chain system typical for $\mathrm{NH}_{4} \mathrm{CuCl}_{3}$, and Sect. IV contains discussion and concluding remarks.

\section{TWO-DIMENSIONAL COUPLED CHAIN MODEL}

Consider a system of zigzag chains coupled in a plane by frustrating zigzag interaction $J^{\prime}$, as shown in Fig. 1. Such a structure is typical, e.g., for $(a c)$-planes of $\mathrm{KCuCl}_{3}$. Let us further assume that the exchange constant $J_{3}$ is antiferromagnetic $(\mathrm{AF})$ and much stronger than all the others, then the system can be viewed as a two-dimensional arrangement of dimers weakly coupled with each other. Assume further that the external magnetic field $\boldsymbol{H}$ is applied in the $z$-direction. The model is described by the Hamiltonian

$$
\begin{aligned}
\widehat{H} & =\sum_{i j}\left\{J_{3} \boldsymbol{S}_{1}^{(i, j)} \cdot \boldsymbol{S}_{2}^{(i, j)}+J_{2} \boldsymbol{S}_{1}^{(i, j)} \cdot \boldsymbol{S}_{2}^{(i-1, j)}\right. \\
& +J_{1}\left(\boldsymbol{S}_{1}^{(i, j)} \cdot \boldsymbol{S}_{1}^{(i+1, j)}+\boldsymbol{S}_{2}^{(i, j)} \cdot \boldsymbol{S}_{2}^{(i+1, j)}\right) \\
& +J^{\prime}\left(\boldsymbol{S}_{2}^{(i, j)} \cdot \boldsymbol{S}_{1}^{(i, j+1)}+\boldsymbol{S}_{2}^{(i, j)} \cdot \boldsymbol{S}_{1}^{(i-1, j+1)}\right) \\
& \left.+g \mu_{B} \boldsymbol{H} \cdot\left(\boldsymbol{S}_{1}^{(i, j)}+\boldsymbol{S}_{2}^{(i, j)}\right)\right\}
\end{aligned}
$$

where $g$ is the Landé factor and $\mu_{B}$ is the Bohr magneton. If one neglects weak interdimer interactions $J_{1}, J_{2}, J^{\prime}$ completely, than the lowest states at each dimer are the singlet $|s\rangle$ and the $S^{z}=+1$ triplet $\left|t_{+}\right\rangle$. We will be interested in the regime of strong fields $g \mu_{B} H \gtrsim J_{3}$, then the other two triplet states $\left|t_{0}\right\rangle,\left|t_{-}\right\rangle$are high in energy and their contribution can be neglected, in spirit of Refs. 9,12,14. In this way one gets a reduced Hilbert space with only two degrees of freedom per dimer, and the problem can be formulated in terms of spinless fermions living on the asymmetric triangular lattice as shown in Fig. 2, with the following effective Hamiltonian $\widehat{\mathcal{H}}$ :

$$
\begin{aligned}
\widehat{\mathcal{H}} & =\mu \sum_{i j} n_{i j}+U \sum_{i j} n_{i j} n_{i+1, j}+t \sum_{i j}\left(c_{i j}^{\dagger} c_{i+1, j}+\text { h.c. }\right) \\
& +U^{\prime} \sum_{i j} n_{i j}\left(n_{i, j+1}+n_{i-1, j+1}\right) \\
& +t^{\prime} \sum_{i j}\left(c_{i j}^{\dagger} c_{i, j+1}+c_{i j}^{\dagger} c_{i-1, j+1}+\text { h.c. }\right) .
\end{aligned}
$$

Here $n_{i j} \equiv c_{i j}^{\dagger} c_{i j}$, and $n_{i j}=1$ corresponds to the $\left|t_{+}\right\rangle$ state on the $(i j)$-th dimer, while absence of particle $\left(n_{i j}=0\right)$ corresponds to the singlet. The magnetization per site in the saturation units $M$ coincides now with the concentration of fermions. The effective interaction constants $U, U^{\prime}$, hopping amplitudes $t, t^{\prime}$, and chemical potential $\mu$ are given by

$$
\begin{aligned}
& U=\left(2 J_{1}+J_{2}\right) / 4, \quad t=\left(2 J_{1}-J_{2}\right) / 4 \\
& U^{\prime}=J^{\prime} / 4, \quad t^{\prime}=-J^{\prime} / 4, \quad \mu=J_{3}-g \mu_{B} H .
\end{aligned}
$$

It is useful to note the particle-hole symmetry of the effective Hamiltonian: written in terms of hole operators, (3) preserves its form (up to a constant shift), with the change only in the value of the chemical potential

$$
\mu \leftrightarrow \mu_{\mathrm{hole}}=-\mu-2 U-4 U^{\prime}
$$

In what follows we assume that the effective interaction constants $U, U^{\prime}$ are positive, i.e. the interaction is repulsive.

\section{A. The "atomic limit"}

Consider first the "atomic limit" of the model (3), i.e. put hopping $t, t^{\prime}$ to zero. Then the problem becomes equivalent to the generalized Ising model in magnetic field, a class of models which is well studied in the theory of alloys (see, e.g., Ref. 21 and references therein). Generally, in this limit the interaction energy per site $E_{\text {int }}$ is a piecewise linear function of the concentration $M$, and the curve $E_{\text {int }}(M)$ has "kinks" (jumps of the first derivative) at certain values of $M$. When the magnetic field increases, the chemical potential $\mu$ becomes negative, and particles start being "pumped" into the system until this process is stopped by the interaction. Kinks in $E_{\text {int }}(M)$ correspond to plateaus in the magnetization curve $M(H)$, and the width of a plateau is proportional to the magnitude of jump in the first derivative. The atomic limit determines the possible number and positions of plateaus; hopping generally tends to smear out the plateaus, and below we will study this effect perturbatively.

In the atomic limit the model (3) is equivalent to the so-called anisotropic triangular nearest-neighbor Ising model in external field, and its ground state is known exactly.22 Up to $M=\frac{1}{3}$ one can insert particles into the system without loosing any interaction energy (see Fig. 2). Thus, 


$$
E_{\text {int }}(M)=0, \quad M \leq \frac{1}{3} .
$$

Using the particle-hole symmetry, one immediately obtains from the above the behavior of $E_{\text {int }}$ above $M=\frac{2}{3}$ :

$$
E_{\text {int }}(M)=\left(2 U+4 U^{\prime}\right)\left(M-\frac{1}{2}\right), \quad M \geq \frac{2}{3} .
$$

In the "intermediate" interval of concentrations $\frac{1}{3}<$ $M<\frac{2}{3}$ the situation depends on the ratio of interaction constants $U$ and $U^{\prime}$. If $U^{\prime}<U$, then the lowest energy cost for inserting new particles above $M=\frac{1}{3}$ is $3 U^{\prime}$ per particle, which is determined by the multiparticle process of inserting at once a row of particles as shown in Fig. 3a. This process leads from the state with $M=\frac{1}{3}$ to the state with $M=\frac{1}{2}$, and another multiparticle process shown in Fig. $3 \mathrm{~b}$ leads from $M=\frac{1}{2}$ to $M=\frac{2}{3}$, taking the energy of $2 U+U^{\prime}$ per particle.

For $U^{\prime}>U$, similar multiparticle processes exist which govern the increase of concentration above $M=\frac{1}{3}$ and $M=\frac{1}{2}$, with the energy cost of $3 U$ and $4 U^{\prime}-U$ per particle, respectively (one can obtain the corresponding pictures by rotating the particle configurations of Fig. 3a,b by $\pi / 3$ but keeping the solid and dotted lines fixed).

Taking all that into account, one obtains the following result for the interaction energy as a function of $M$ in the $\left[\frac{1}{3}, \frac{2}{3}\right]$ interval:

$$
E_{\text {int }}= \begin{cases}3 U^{\prime}\left(M-\frac{1}{3}\right), & \frac{1}{3}<M \leq \frac{1}{2} \\ \left(2 U+U^{\prime}\right) M-U, & \frac{1}{2}<M<\frac{2}{3}\end{cases}
$$

for $U^{\prime}<U$, and

$$
E_{\mathrm{int}}= \begin{cases}3 U\left(M-\frac{1}{3}\right), & \frac{1}{3}<M \leq \frac{1}{2} \\ \left(4 U^{\prime}-U\right)\left(M-\frac{1}{2}\right)-\frac{U}{2}, & \frac{1}{2}<M<\frac{2}{3}\end{cases}
$$

for $U^{\prime}>U$. The resulting behavior of $E_{\mathrm{int}}(M)$ is schematically shown in Fig. 1 . Thus, generally three plateaus are possible, at $M=\frac{1}{3}, \frac{1}{2}$, and $\frac{2}{3}$. In the limit of zero interchain interaction $U^{\prime}=0$ only the plateau at $M=\frac{1}{2}$ survives, so that one may think of this plateau as coming from single chains, while plateaus at $\frac{1}{3}$ and $\frac{2}{3}$ appear due to $2 \mathrm{D}$ interchain coupling. The plateau at $M=\frac{1}{2}$ disappears when 2D interactions become isotropic (i.e., $U^{\prime}=U$ ).

It should be remarked that the positions of those "additional" plateaus strongly depend on the topology of the lattice. For instance, if one considers a trellis lattice shown in Fig. 河 (this type of interchain coupling is realized, e.g., in $\mathrm{Sr}_{n-1} \mathrm{Cu}_{n+1} \mathrm{O}_{2 n}$ family 23 ), then spinless fermions live not on a triangular, but on a square effective lattice, and the only possible plateau would be at $M=\frac{1}{2}$.

\section{B. The effect of hopping}

Since in absence of hopping we know the solution exactly, it is natural to try to take hopping into account perturbatively. Then one can determine the critical fields marking the beginning and the end of each plateau.

To justify the use of perturbative approach with hopping term as a perturbation, one has to demand that $J_{2}$ is close to $2 J_{1}$, i.e. that a single zigzag chain is close to the Shastry-Sutherland line,24 and that the interchain coupling is much smaller than $J_{1}$ :

$$
\left|2 J_{1}-J_{2}\right| \ll J_{1}, \quad J^{\prime} \ll J_{1} .
$$

Comparing (7) with (4), one can see that it does not make sense to consider the case $U^{\prime}>U$, and in what follows we will assume that $U^{\prime} \ll U$.

Let us start with the $M=\frac{1}{3}$ structure shown in Fig. 2, where one third of the total number of sites $N$ is occupied by particles. Increasing of the magnetization from $M=$ $\frac{1}{3}$ is determined by the multiparticle process of inserting a row of $L$ new particles, $L \gg 1$ (see Fig. 3 a). Then there are no first-order corrections to $E_{N / 3+L}$, and in the second order the gap $\Delta_{c 3}=\left(E_{N / 3+L}-E_{N / 3}\right) / L$ reads as

$$
\begin{aligned}
\Delta_{c 3}^{U^{\prime}<U} & =\mu+3 U^{\prime}+\frac{4 t^{\prime 2}}{2 U-U^{\prime}}+\frac{4 t^{2}}{U}-\frac{t^{2}}{U^{\prime}} \\
& -\frac{2 t^{2}-12 t^{\prime 2}}{U+U^{\prime}}+\frac{5 t^{\prime 2}}{U}+O(1 / L) .
\end{aligned}
$$

Zero of the gap $\Delta_{c 3}$ gives the critical field $H_{c 3}$.

Similarly, for $U^{\prime}<U$, increase of the magnetization from the $M=\frac{1}{2}$ state is determined by the multiparticle process shown in Fig. $3 \mathrm{~b}$, which, up to the second order, yields the following expression for the gap $\Delta_{c 5}=\left(E_{N / 2+L}-E_{N / 2}\right) / L$ :

$$
\begin{aligned}
\Delta_{c 5} & =\mu+2 U+U^{\prime}+\left(4 t^{\prime 2}-3 t^{2}\right) / U+4 t^{\prime 2} /\left(U+U^{\prime}\right) \\
& +3 t^{2} / U^{\prime}-6 t^{\prime 2} /\left(2 U-U^{\prime}\right)+O(1 / L) .
\end{aligned}
$$

To obtain the critical field $H_{c 2}$, one has to consider the set of degenerate states with one particle taken out of the structure shown in Fig. 2; the degeneracy is lifted only in the second order, giving the following dispersion $\varepsilon_{c 2}(\boldsymbol{k})=E_{N / 3-1}(\boldsymbol{k})-E_{N / 3}$ of the $N / 3-1$ excitation

$$
\begin{aligned}
\varepsilon_{c 2}(\boldsymbol{k}) & =-\mu+\frac{8}{U^{\prime}}\left[t^{2}+t^{2}+t t^{\prime}\left(\cos k_{1}+\cos k_{2}\right)\right] \\
& +\frac{8 t^{\prime 2}}{U}\left[1+\cos \left(k_{1}-k_{2}\right)\right]-\frac{3 t^{2}}{U^{\prime}}-\frac{12 t^{\prime 2}}{U+U^{\prime}}
\end{aligned}
$$

and the gap $\Delta_{c 2}=\min \varepsilon_{c 2}(\boldsymbol{k})$ is given by

$$
\begin{aligned}
\Delta_{c 2} & =-\mu-3 t^{2} / U^{\prime}-12 t^{\prime 2} /\left(U+U^{\prime}\right) \\
& +8\left(|t|-\left|t^{\prime}\right|\right)^{2} / U^{\prime}+16 t^{\prime 2} / U, \quad\left|t / t^{\prime}\right|>2 U^{\prime} / U, \\
\Delta_{c 2} & =-\mu+5 t^{2} / U^{\prime}-12 t^{\prime 2} /\left(U+U^{\prime}\right) \\
& -4 t^{2} U / U^{\prime 2}+8 t^{\prime 2} / U^{\prime}, \quad\left|t / t^{\prime}\right|<2 U^{\prime} / U .
\end{aligned}
$$

Finally, it is easy to calculate the value of the first critical field $H_{c 1}$ which indicates the point where the injection of triplets starts. 14 In the vicinity of $H_{c 1}$ the density of 
triplets is very low, so that one can just neglect the interaction terms in (3). The hopping part of the effective Hamiltonian yields the dispersion $E_{1}-E_{0} \equiv \varepsilon(\boldsymbol{k})$,

$$
\varepsilon(\boldsymbol{k})=\mu+2 t \cos k_{1}+2 t^{\prime}\left\{\cos k_{2}+\cos \left(k_{1}-k_{2}\right)\right\} .
$$

The gap $\Delta_{c 1}=\min \varepsilon(\boldsymbol{k})$ is given by

$$
\mu_{c 1}=\left\{\begin{array}{ll}
\mu-2 t-\left(t^{\prime}\right)^{2} / t & \text { at } t>0 \\
\mu+2 t-4\left|t^{\prime}\right| & \text { at } t<0
\end{array} .\right.
$$

All the other critical fields now can be obtained by exploiting the particle-hole symmetry (5), which connects the following pairs:

$$
\begin{aligned}
& \mu_{S} \leftrightarrow \mu_{c 1}, \mu_{c 5} \leftrightarrow \mu_{c 4}, \\
& \mu_{c 3} \leftrightarrow \mu_{c 6}, \quad \mu_{c 2} \leftrightarrow \mu_{c 7} .
\end{aligned}
$$

Zeros of the gaps given by the formulas (8), (9), (11), (12), together with the definition of $\mu$ as stated in (4), determine the critical fields. It should be remarked that in the derivation of (12) we did not use perturbation theory, so that the expression for $H_{c 1}$ is exact within the effective model (3). It can be improved, however, by including the processes involving the other two triplet states which were discarded in our consideration, in spirit of the degenerate perturbation theory (see, e.g., Ref. 25).

\section{THREE-DIMENSIONAL $\mathrm{NH}_{4} \mathrm{CuCl}_{3}$-TYPE LATTICE}

Now let us proceed to a more complicated model, which could be useful for understanding the physics of magnetization process of $\mathrm{NH}_{4} \mathrm{CuCl}_{3}$. Consider a threedimensional model which can be imagined as set of twodimensional systems ("layers") from Section II stacked on top of each other, as shown schematically in Fig. 7 . Besides exchange interactions $J_{1,2,3}, J^{\prime}$ inside a single layer, we have now two additional interlayer interaction constants $J^{\prime \prime}, J^{\prime \prime \prime}$. This mel corresponds to the lattice structure of $\mathrm{NH}_{4} \mathrm{CuCl}_{3}, 1617$ with only nearest-neighbor exchange couplings taken into account. As before, we assume that all interactions are antiferromagnetic, and that $J_{3}$ is much stronger than the other exchange constants, so that the system can be viewed as a set of weakly coupled dimers. Along the same lines of reasoning as those used in the previous Section, one obtains the effective spinless fermion model shown pictorially in Fig. 8; in addition to (踹), there are now two more pairs of interaction and hopping constants,

$$
U^{\prime \prime}=\frac{1}{2} J^{\prime \prime}, \quad t^{\prime \prime}=0, \quad U^{\prime \prime \prime}=\frac{1}{4} J^{\prime \prime \prime}=-t^{\prime \prime \prime} .
$$

There is again a particle-hole symmetry; the relation (5) now has to be modified to

$$
\mu \leftrightarrow \mu_{\text {hole }}=-\mu-2 U-4 U^{\prime}-4 U^{\prime \prime}-4 U^{\prime \prime \prime} .
$$

One can find that up to the concentration $M=\frac{1}{4}$ particles can be pumped into the system without interacting with each other. The corresponding structure with $M=\frac{1}{4}$ is shown in Fig. 8 8 a; it is easy to see that no new particles can be inserted without interaction. Thus, the interaction energy $E_{\text {int }}$ as a function of $M$ always has a kink at $M=\frac{1}{4}$, and a symmetrical kink should be present at $M=\frac{3}{4}$. Those are exactly the values of $M$ at which the magnetization plateaus in $\mathrm{NH}_{4} \mathrm{CuCl}_{3}$ are observed.15 However, it can be shown that in the atomic limit there are always other kinks at different values of $M$ present in $E_{\text {int }}(M)$. Simply comparing the energies of different structures and displaying them in the $E_{\text {int }}(M)$ plot, one can see that there always exists certain $M=\frac{1}{2}$ structure whose energy is below $E_{\text {int }}\left(\frac{1}{4}\right)+E_{\text {int }}\left(\frac{3}{4}\right)$, i.e., the corresponding point in the $E_{\text {int }}(M)$ plot lies below the line connecting the $M=\frac{1}{4}$ and $M=\frac{3}{4}$ structures; in the same way one can check that $M=\frac{1}{3}$ and $M=\frac{2}{3}$ structures can be also favored under certain conditions on the model parameters.

Since no other plateaus except $M=\frac{1}{4}$ and $M=\frac{3}{4}$ are observed in $\mathrm{NH}_{4} \mathrm{CuCl}_{3}$, this presents a problem. Let us consider a particular regime with the exchange constants satisfying the following inequalities:

$$
\begin{aligned}
& J^{\prime \prime \prime} \leq J^{\prime \prime}, \quad J^{\prime} \leq 2 J^{\prime \prime \prime} \\
& J^{\prime} \leq 2 J_{1}+J_{2}+4\left(J^{\prime \prime}-J^{\prime \prime \prime}\right) .
\end{aligned}
$$

Then one can show that in the atomic limit the only additional plateau structure realized in between $M=$ $\frac{1}{4}$ and $M=\frac{3}{4}$ is the $M=\frac{1}{2}$ structure shown in Fig. 8 b. Then, generally, there exist eight critical fields, as in the $2 \mathrm{D}$ problem considered in the previous section, which can be calculated perturbatively in the same way. The perturbation theory results in three dimensions are somewhat cumbersome even in the second order, so that they are listed in the Appendix.

The important point is that in the regime determined by (15) hopping reduces the width of the $M=\frac{1}{2}$ plateau already in the first order, while corrections to the width of $M=\frac{1}{4}, \frac{3}{4}$ plateaus start from the second order. Thus, in the above regime there is only one additional plateau at $M=\frac{1}{2}$ which is smeared by hopping more effectively than the plateaus at $M=\frac{1}{4}, \frac{3}{4}$. This could be a qualitative explanation of the real situation in $\mathrm{NH}_{4} \mathrm{CuCl}_{3}$, if one assumes that the additional plateau is completely wiped out.

\section{DISCUSSION AND SUMMARY}

In this paper I show that in quasi-one-dimensional spin systems the location of plateaus in the dependence of magnetization on applied field can be considerably affected by presence of two- or three-dimensional interchain couplings. As an example, a simple model of twodimensionally coupled zigzag chains is considered in a 
dimer limit. It has been shown that, depending on the lattice structure and on the interplay between the model parameters, the system can exhibit plateaus at $\frac{1}{3}$ and $\frac{2}{3}$ of the saturation, in addition to the "usual" plateau at one half of the saturation determined by physics of a single chain.

We argue that this mechanism can be relevant for the recept intriguing high-field magnetization measurements 15 in $\mathrm{NH}_{4} \mathrm{CuCl}_{3}$, since the threedimensional lattice structure of this material naturally favors the appearance of plateaus at $\frac{1}{4}$ and $\frac{3}{4}$ of the saturation, i.e., exactly at those positions where the plateaus were actually observed.

Unfortunately, the arguments presented here cannot be used for any quantitative predictions concerning the exchange constants in $\mathrm{NH}_{4} \mathrm{CuCl}_{3}$, because of the following reasons: (i) zero-field magnetic ground state of $\mathrm{NH}_{4} \mathrm{CuCl}_{3}$ implies that the first critical field $H_{c 1} \leq 0$, and from A8 one can clearly see that then $J_{3}$ cannot be much stronger than all the other couplings, as we assumed; (ii) to justify considering the hopping term as a perturbation, one would need to satisfy (7), and the condition $J_{2} \rightarrow 2 J_{1}$ does not seem playsjble on the basis of the available crystal structure data.17

\section{ACKNOWLEDGMENTS}

I am deeply grateful to H.-J. Mikeska for bringing the problem of plateaus in $\mathrm{NH}_{4} \mathrm{CuCl}_{3}$ to my attention and for subsequent active interest in the work. It is also my pleasure to thank W. Selke and H. Tanaka for useful comments. The hospitality of Hannover Institute for Theoretical Physics is gratefully acknowledged. This work was supported by the German Ministry for Research and Technology (BMBF) under the contract 03MI4HAN8 and by the Ukrainian Ministry of Science (grant 2.4/27).

\section{APPENDIX: GAPS IN 3D MODEL}

In the 3D model of Sect. III the magnetization curve looks similarly to that presented in Fig. 6, except that now we have plateaus at $\frac{1}{4}$ and $\frac{3}{4}$ instead $\frac{1}{3}$ and $\frac{2}{3}$, respectively. The effect of hopping can be considered perturbatively, provided that (7) is satisfied together with the additional inequalities

$$
J^{\prime \prime} \ll J_{1}, \quad J^{\prime \prime \prime} \ll J_{1} .
$$

In the second order of perturbation theory, the dispersion $\varepsilon_{c 3}(\boldsymbol{k})$ of the excitation with one extra particle inserted above the $M=\frac{1}{4}$ state, and the dispersion $\varepsilon_{c 2}(\boldsymbol{k})$ of the excitation resulting from taking away one particle off the same $M=\frac{1}{4}$ state, can be represented in the following form

$$
\begin{aligned}
\varepsilon_{c, i}(\boldsymbol{k}) & =\varepsilon_{c, i}^{0}+A_{i} \cos \left(k_{1}+k_{2}\right)+B_{i} \cos 2\left(k_{1}-k_{2}\right) \\
& +2 C_{i} \cos \left(k_{1}-k_{2}\right)\left[\cos k_{3}+\cos \left(k_{1}+k_{2}-k_{3}\right)\right],
\end{aligned}
$$

There are no first-order corrections to the energies of $N / 4 \pm 1$ states. The corresponding constants for the $N / 4+1$ excitation are given by the formulas

$$
\begin{aligned}
\varepsilon_{c 3}^{0} & =\mu+2 U^{\prime}+4 U^{\prime \prime \prime}+\frac{t^{2}}{U^{\prime \prime}}+\frac{t^{2}}{U^{\prime \prime \prime}}+\frac{2 t^{2}}{U^{\prime}+2 U^{\prime \prime}} \\
& +\frac{6\left(t^{2}+t^{2}\right)}{U-U^{\prime}+2 U^{\prime \prime}-4 U^{\prime \prime \prime}}+\frac{16\left[t^{2}+\left(t^{\prime \prime \prime}\right)^{2}\right]}{U^{\prime}+3 U^{\prime \prime \prime}} \\
& -\frac{12 t^{2}}{U^{\prime}+4 U^{\prime \prime \prime}}+\frac{2 t^{\prime 2}}{2 U-U^{\prime}+2 U^{\prime \prime}}+\frac{2 t^{2}}{U+U^{\prime}+2 U^{\prime \prime}} \\
& -\frac{12 t^{\prime 2}}{U+2 U^{\prime \prime}}-\frac{12 t^{2}}{U^{\prime}+2 U^{\prime \prime}}+\frac{4 t^{\prime 2}}{U+3 U^{\prime \prime}-U^{\prime \prime \prime}} \quad(\mathrm{A} 3 \\
& +\frac{4 t^{2}}{U^{\prime}+3 U^{\prime \prime}-U^{\prime \prime \prime}}+\frac{4 t^{2}}{U^{\prime}+2 U^{\prime \prime}-U^{\prime \prime \prime}}+\frac{4\left(t^{\prime \prime \prime}\right)^{2}}{U^{\prime}+U^{\prime \prime \prime}} \\
& +\frac{4 t^{\prime 2}}{U+2 U^{\prime \prime}-U^{\prime \prime \prime}}+\frac{16\left(t^{\prime \prime \prime}\right)^{2}}{U^{\prime 2}-U^{\prime}+2 U^{\prime \prime}}-\frac{4\left(t^{\prime \prime \prime}\right)^{2}}{2 U^{\prime}+3 U^{\prime \prime \prime}}, \\
A_{3} & =\frac{8 t t^{\prime}}{U-U^{\prime}+2 U^{\prime \prime}-4 U^{\prime \prime \prime}}+\frac{4 t^{\prime} t^{\prime \prime \prime}}{U^{\prime}+U^{\prime \prime \prime}}, \\
B_{3} & =\frac{t^{\prime 2}}{U^{\prime \prime \prime}}, \quad C_{3}=\frac{U^{\prime}+3 U^{\prime \prime \prime}}{U^{\prime}}
\end{aligned}
$$

and for the $N / 4-1$ excitation one obtains

$$
\begin{aligned}
\varepsilon_{c 2}^{0} & =-\mu+\frac{8\left(t^{\prime \prime \prime}\right)^{2}}{U^{\prime}}+\frac{2\left(t^{2}+t^{2}\right)}{U^{\prime \prime}}+\frac{2 t^{\prime 2}}{U^{\prime \prime \prime}}+\frac{2 t^{2}}{U^{\prime}+U^{\prime \prime}} \\
& +\frac{2 t^{\prime 2}}{U+U^{\prime \prime}}+\frac{16\left[t^{\prime 2}+\left(t^{\prime \prime \prime}\right)^{2}\right]}{U^{\prime}+3 U^{\prime \prime \prime}}-\frac{6 t^{\prime 2}}{U+2 U^{\prime \prime}} \quad(\mathrm{A} 5) \\
& -\frac{12 t^{\prime 2}}{U^{\prime}+4 U^{\prime \prime \prime}}-\frac{14\left(t^{\prime \prime \prime}\right)^{2}}{2 U^{\prime}+3 U^{\prime \prime \prime}}-\frac{6 t^{2}}{U^{\prime}+2 U^{\prime \prime}}, \\
A_{2} & =\frac{4 t t^{\prime}}{U^{\prime \prime}}+\frac{4\left(t^{\prime \prime \prime}\right)^{2}}{U^{\prime}}, \quad B_{2}=\frac{t^{\prime 2}}{U^{\prime \prime \prime}}, \quad C_{2}=\frac{8 t^{\prime} t^{\prime \prime \prime}}{U^{\prime}+3 U^{\prime \prime \prime}} .
\end{aligned}
$$

Closing of the gaps $\Delta_{c, i}=\min \varepsilon_{c, i}(\boldsymbol{k})(i=2,3)$ determines the critical fields $H_{c 2}, H_{c 3}$ marking the beginning and the end of the $M=\frac{1}{4}$ plateau, respectively. Taking into account that $B_{2,3}$ and $C_{2,3}$ are always positive [by our assumption on antiferromagnetic character of all exchange interactions, see (4), (13)], one can obtain the following formulas for the gaps:

$$
\Delta_{c}=\varepsilon_{c}^{0}+\left\{\begin{array}{ll}
A+B-4 C, & B<C \\
A-B-2 C^{2} / B, & B>C
\end{array} \quad \text { at } A<0,\right.
$$

and for $A>0$ the result is a bit more complicated,

$$
\Delta_{c}=\varepsilon_{c}^{0}+\left\{\begin{array}{ll}
-A-B, & C<(A B)^{1 / 2} \\
A+B-4 C, & A<C, B<C \\
B-A-2 C^{2} / A, & (A B)^{1 / 2}<C<A \\
A-B-2 C^{2} / B, & (A B)^{1 / 2}<C<B
\end{array} .\right.
$$

[We have omitted the subscripts 2,3 above for the sake of clarity]. One can see that the expressions for $\Delta_{c 2}$, $\Delta_{c 3}$ "explode" when either of the interchain interaction 
constants $U^{\prime}, U^{\prime \prime}, U^{\prime \prime \prime}$ vanishes; this corresponds to the obvious fact that the $M=\frac{1}{4}$ plateau ceases to exist if any of the interchain interactions is absent.

The energy $\varepsilon_{N / 2+1}(\boldsymbol{k})=\varepsilon_{N / 2+1}^{(1)}(\boldsymbol{k})+\varepsilon_{N / 2+1}^{(2)}(\boldsymbol{k})$ of the excitation with one extra particle above the $M=\frac{1}{2}$ state contains both first- and second-order corrections. In the first order approximation one obtains

$$
\begin{aligned}
\varepsilon_{N / 2+1}^{(1)} & =\mu+2\left(U+U^{\prime}+2 U^{\prime \prime}\right) \\
& +2 t^{\prime} \cos \left(k_{1}-k_{2}\right)+2 t^{\prime \prime \prime}\left[\cos k_{3}+\cos \left(k_{1}+k_{3}\right)\right],
\end{aligned}
$$

and the second-order contribution is

$$
\begin{aligned}
\varepsilon_{N / 2+1}^{(2)} & =\frac{t^{2}\left(1+\cos 2 k_{1}\right)}{U^{\prime \prime}-U^{\prime \prime \prime}}+\frac{2 t^{\prime 2}\left(1+\cos 2 k_{2}\right)}{U-U^{\prime}+2\left(U^{\prime \prime}-U^{\prime \prime \prime}\right)} \\
& +\frac{4 t^{2}+4 t^{2}+8 t t^{\prime} \cos \left(k_{1}+k_{2}\right)}{U-U^{\prime}+4\left(U^{\prime \prime}-U^{\prime \prime \prime}\right)} \\
& +\frac{4 t^{2}+4 t^{\prime 2}+8 t t^{\prime} \cos \left(k_{1}-k_{2}\right)}{U+4\left(U^{\prime \prime}-U^{\prime \prime \prime}\right)} \\
& -\frac{22 t^{2}}{U+4\left(U^{\prime \prime}-U^{\prime \prime \prime}\right)}-\frac{22 t^{\prime 2}}{2 U-U^{\prime}+4\left(U^{\prime \prime}-U^{\prime \prime \prime}\right)} \\
& +\frac{4 t^{\prime 2}}{2 U-U^{\prime}+3\left(U^{\prime \prime}-U^{\prime \prime \prime}\right)}+\frac{4 t^{2}}{U+3\left(U^{\prime \prime}-U^{\prime \prime \prime}\right)} \\
& +\frac{4 t^{\prime 2}}{2 U-U^{\prime}+3 U^{\prime \prime}-4 U^{\prime \prime \prime}}+\frac{4 t^{2}}{U+4 U^{\prime \prime}-3 U^{\prime \prime \prime}} \\
& +\frac{4 t^{2}}{U+3 U^{\prime \prime}-4 U^{\prime \prime \prime}}+\frac{4 t^{2}}{2 U-U^{\prime}+4 U^{\prime \prime}-3 U^{\prime \prime \prime}} \\
& +\frac{2 t^{2}}{U+U^{\prime}+4\left(U^{\prime \prime}-U^{\prime \prime \prime}\right)}+\frac{2 t^{\prime 2}}{2 U+4\left(U^{\prime \prime}-U^{\prime \prime \prime}\right)} .
\end{aligned}
$$

Zero of the corresponding gap $\Delta_{c 5}=\min \varepsilon_{N / 2+1}(\boldsymbol{k})$, together with the formulas (何), 13, determines the critical field $H_{c 5}$ marking the end of the $M=\frac{1}{2}$ plateau.

The energy $\varepsilon_{c 1}(\boldsymbol{k})$ of the one-particle excitation above the non-magnetic $(M=0)$ state is completely determined by the hopping part of the Hamiltonian and has the form

$$
\begin{aligned}
\varepsilon_{c 1}(\boldsymbol{k}) & =\mu+2 t \cos k_{1}+2 t^{\prime}\left[\cos k_{2}+\cos \left(k_{1}-k_{2}\right)\right] \\
& +2 t^{\prime \prime \prime}\left[\cos k_{3}+\cos \left(k_{1}+k_{2}-k_{3}\right)\right]
\end{aligned}
$$

the gap in the above dispersion closes at the critical field $H_{c 1}$ above which the magnetization starts to increase from zero.

Exploiting the particle-hole symmetry (14) allows one to connect the following pairs of critical fields:

$$
\begin{aligned}
H_{S} & \leftrightarrow H_{c 1}, \quad H_{c 5} \leftrightarrow H_{c 4}, \\
H_{c 3} & \leftrightarrow H_{c 6}, \quad H_{c 2} \leftrightarrow H_{c 7} .
\end{aligned}
$$

Setting $H_{c 4}$ equal to $H_{c 5}$, we can obtain a condition for "closing" the $M=\frac{1}{2}$ plateau; restricting ourselves to the first order expressions for the sake of simplicity, we get

$$
J_{1}+\frac{1}{2} J_{2}+J^{\prime \prime} \leq J^{\prime}+3 J^{\prime \prime \prime}
$$

However, it is easy to see that (A10) is already outside the range of validity of the perturbation theory determined by (7), (A1), so that this condition should be understood only as a rough estimate indicating that $J^{\prime}, J^{\prime \prime \prime}$ have to be sufficiently strong to wipe out the plateau at $M=\frac{1}{2}$.

* Permanent address: Institute of Magnetism, 36(b) Vernadskii av., 252142 Kiev, Ukraine; email address: kolezhuk@joanna.ru.kiev.ua

${ }^{1}$ K. Hida, J. Phys. Soc. Jpn. 63, 2359 (1994).

${ }^{2}$ K. Okamoto, Solid State Commun. 98, 245 (1996).

${ }^{3}$ T. Tonegawa, T. Nakao, and M. Kaburagi, J. Phys. Soc. Jpn. 65, 3317 (1996).

${ }^{4}$ M. Oshikawa, M. Yamanaka, and I. Affleck, Phys. Rev. Lett. 78, 1984 (1997).

${ }^{5}$ K. Totsuka, Phys. Lett. A228, 103 (1997).

${ }^{6}$ D. C. Cabra, A. Honecker, and P. Pujol, Phys. Rev. Lett. 79, 5126 (1997); see also e-print cond-mat/9802035.

${ }^{7}$ T. Tonegawa, T. Nishida, and M. Kaburagi, in Proc. of the 5th Int. Conf. on Research in High Magnetic Fields, Sydney, 1997 (to appear in Physica B, 1998).

${ }^{8}$ Y. Narumi, M. Hagiwara, R. Sato, K. Kindo, H. Nakano, and M. Takahashi, in Proc. of the 5th Int. Conf. on Research in High Magnetic Fields, Sydney, 1997 (to appear in Physica B, 1998).

${ }^{9}$ K. Totsuka, Phys. Rev. B 57, 3454 (1998).

${ }^{10}$ H. Nakano and M. Takahashi, J. Phys. Soc. Jpn. 67, 1126 (1998).

11 T. Sakai and M. Takahashi, Phys. Rev. B 57, R3201 (1998).

${ }^{12}$ K. Totsuka, to appear in EPJ B, June 1998.

${ }^{13}$ D. C. Cabra and M. D. Grynberg, e-print condmat/9803368.

${ }^{14}$ F. Mila, e-print cond-mat/9805029.

${ }^{15}$ W. Shiramura, K. Takatsu, B. Kurniawan, H. Tanaka, H. Uekusa, Y. Ohashi, K. Takizawa, H. Mitamura, and T. Goto, J. Phys. Soc. Jpn. 67, 1548 (1998).

${ }^{16}$ H. Tanaka, K. Takatsu, W. Shiramura, and T. Ono, J. Phys. Soc. Jpn. 65, 1945 (1996).

${ }^{17}$ K. Takatsu, W. Shiramura, and H. Tanaka, J. Phys. Soc. Jpn. 66, 1611 (1997).

18 T. Nakamura and K. Okamoto, e-print cond-mat/9709295.

19 Those arguments, however, have to be taken with caution since they are obtained by fitting in a purely onedimensional model.

20 T. Kato, K. Takatsu, H. Tanaka, W. Shiramura, M. Mori, K. Nakajima, and K. Kakurai, J. Phys. Soc. Jpn. 67, 752 (1998).

${ }^{21}$ F. Ducastelle, Order amd Phase Stability in Alloys (NorthHolland, Amsterdam, 1991), Chapter 3.

${ }^{22}$ K. Y. Lin and F. Y. Wu, Z. Phys. B 33, 181 (1979).

${ }^{23}$ M. Takano, Z. Hiroi, M. Azuma, and Y. Takeda, Jpn. J. Appl. Phys. 7, 3 (1992); Z. Hiroi, M. Azuma, M. Takano, 
and Y. Bando, J. Solid State Chem. 95, 230 (1991).

${ }^{24}$ B. S. Shastry and B. Sutherland, Phys. Rev. Lett. 47, 964 (1981).

${ }^{25}$ Kunj Tandon, Siddhartha Lal, Swapan K. Pati, S. Ramasesha, Diptiman Sen, e-print cond-mat/9806111.

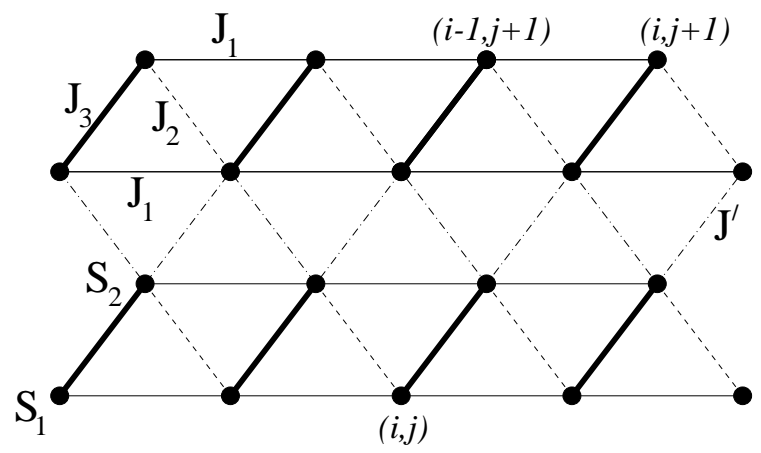

FIG. 1. Two-dimensional system of coupled zigzag chains described by the Hamiltonian (2), a structure which is rodized in the $(a c)$-planes of the $\mathrm{KCuCl}_{3}$ family compounds 16.17

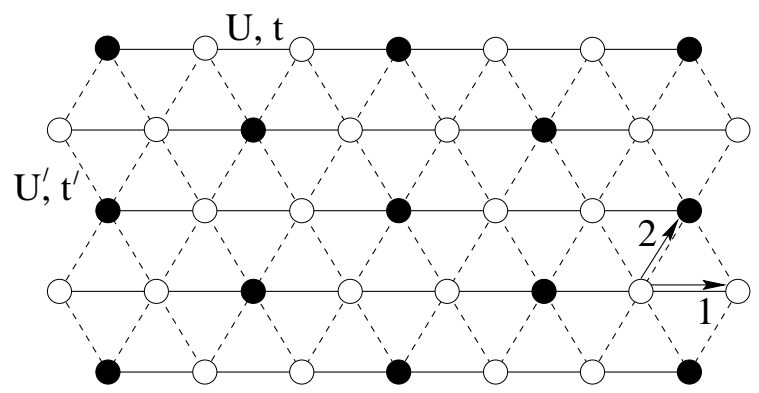

FIG. 2. Effective model of spinless fermions on a triangular lattice resulting from the system shown in Fig. 1, as described by (3). The structure with $M=\frac{1}{3}$ in the "atomic limit" is shown; open and filled circles denote respectively empty and filled sites (i.e., dimers in $|s\rangle$ and $\left|t_{+}\right\rangle$states). 


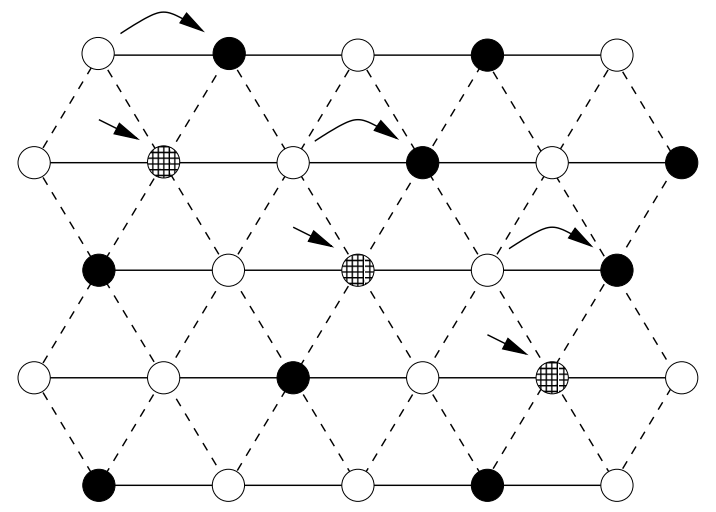

(a)



FIG. 3. Different processes of increasing the concentration in the atomic limit of the model (3) at $U^{\prime}<U$ : (a) the process leading from $M=\frac{1}{3}$ to the intermediate plateau at $M=\frac{1}{2}$; (b) the process increasing the magnetization above $M=\frac{1}{2}$. Half-filled and black circles represent respectively 'new' particles inserted into the system and 'old' ones which formed the $M=\frac{1}{3}$ or $M=\frac{1}{2}$ structure.

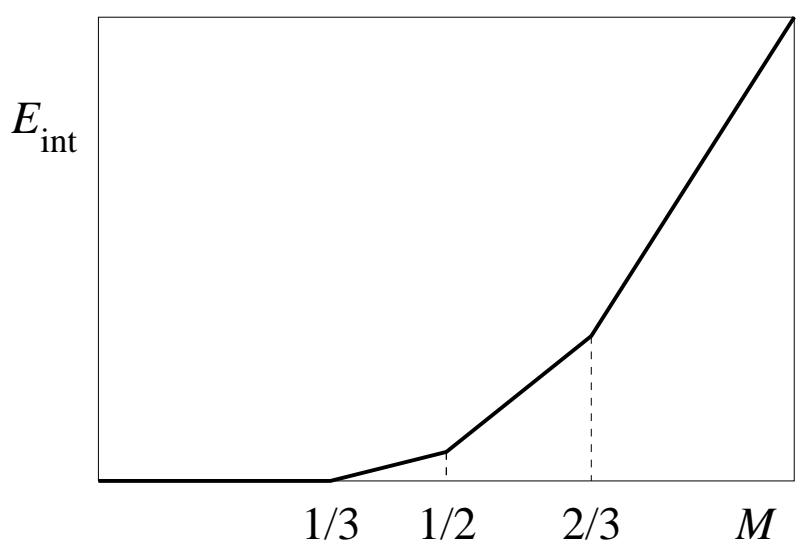

FIG. 4. A schematic look of the interaction energy per site $E_{\text {int }}$ as a function of the concentration $M$, in the "atomic limit" of (3).

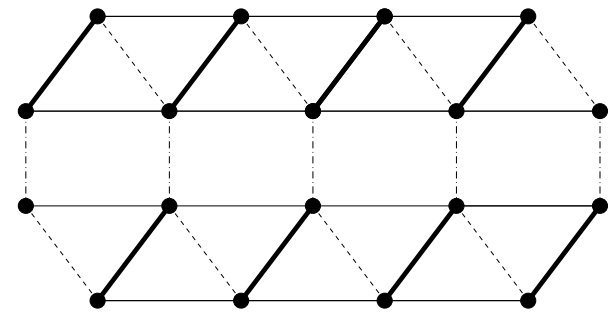

FIG. 5. Dimers coupled in a trellis lattice. This topology allows only one plateau at $M=\frac{1}{2}$.

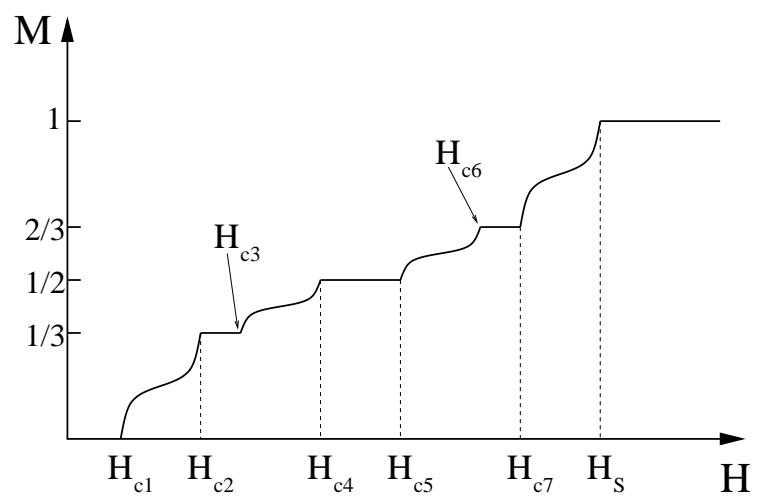

FIG. 6. Schematic plot of the magnetization $M$ as a function of the applied field $H$ for the effective model (3). Solid and dashed lines show the magnetization curves for the cases $U^{\prime} \geq U$ and $U^{\prime}<U$, respectively.
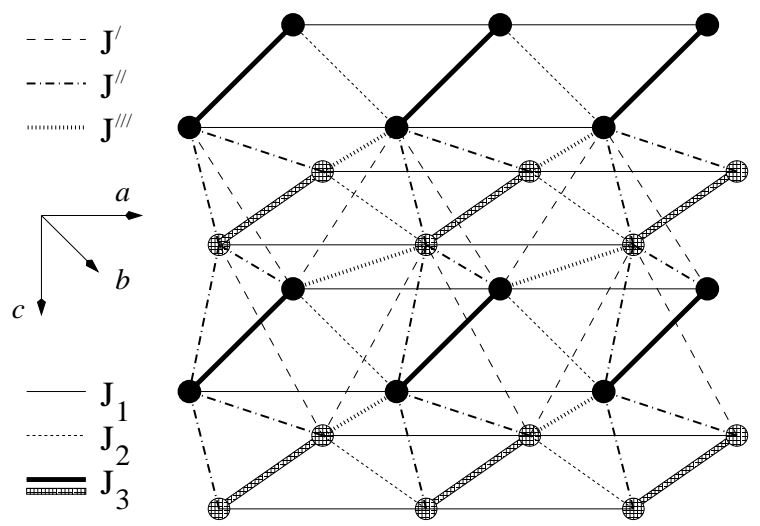

FIG. 7. Three-dimprimally coupled spin structure realized in $\mathrm{KCuCl}_{3}$ family. 16 . 17 Filled circles denote $S=\frac{1}{2}$ spins of the lower layer in the (ac)-plane, and half-filled circles represent spins of the upper layer. 

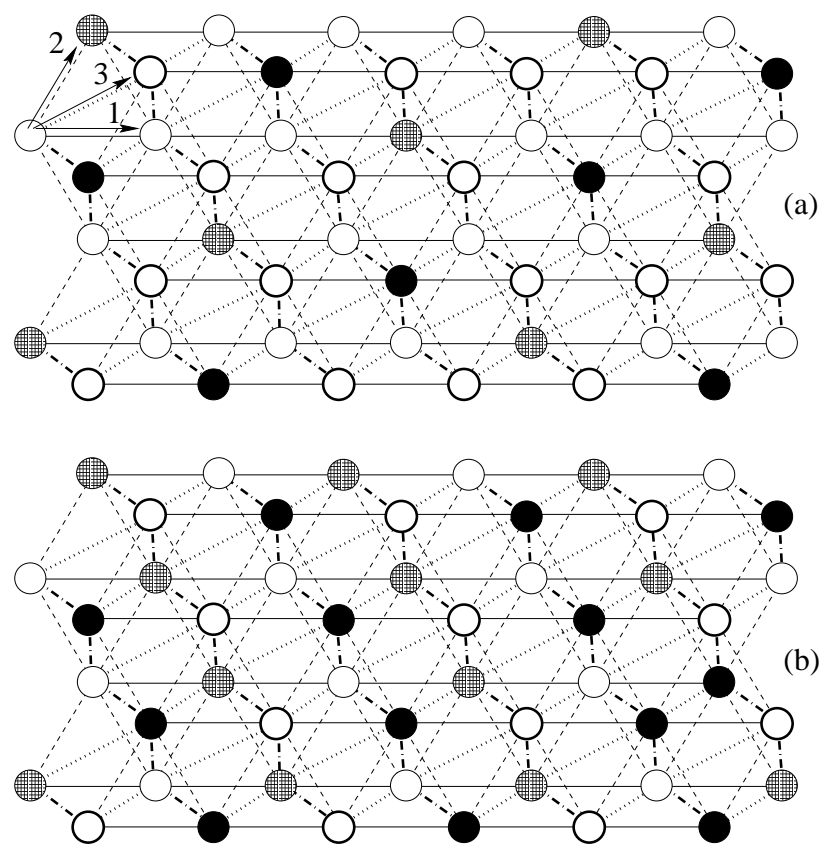

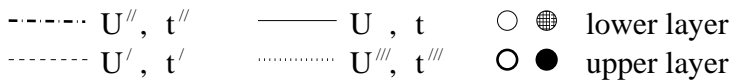

FIG. 8. Visual representation of the effective spinless fermion model resulting from the model shown in Fig. 7; the interaction constants $U$ and hopping amplitudes $t$ are indicated for each link and are given by the formulae (4), (13). Filled and empty circles represent occupied and empty sites; (a) the $M=\frac{1}{4}$ structure; (b) the $M=\frac{1}{2}$ structure which is realized under conditions (15). 